Karina de SÁ AdAmi Gonçalves Brandão ${ }^{1}$

Bruno Gil de Carvalho Lima ${ }^{1}$

Ana Gabriela Álvares Travassos ${ }^{2}$

Fabielle de Olivelra Rocha de Brito ${ }^{3}$

Eveline Xavier Pereira de Souza ${ }^{3}$

TATIANA HAGUiHARA ${ }^{4}$

Carlos Alberto Lima da Silva

\title{
Adesão à dupla contracepção entre mulheres infectadas pelo HIV
}

\section{Dual contraception adberence among HIV-infected women}

Artigo Original

Palavras-chave

HIV

Anticoncepção

Preservativos

Acetato de medroxiprogesterona

Keywords

HIV

Contraception

Condoms

Medroxyprogesterone acetate

\section{Resumo}

OBJETIVO: Verificar a adesão à dupla contracepção entre mulheres infectadas pelo HIV usando acetato de medroxiprogesterona de depósito (AMPD) e condom. MÉTODOS: Corte transversal realizado em centro de referência de dezembro 2013 a setembro 2014. Entrevistadas 114 mulheres HIV (+), 15 a 49 anos, em uso de AMPD e condom para contracepção, aplicando questionário clínico epidemiológico, construído após painel Delphi e validação de

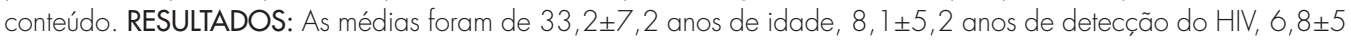
anos de uso de terapia antirretroviral (TARV) e 737,6 $6 \pm 341,1$ células CD4/ $\mathrm{mm}^{3}$. Adquiriram HIV pelo sexo 98,2\% (1 $12 / 1$ 14). Identificadas 85,9\% (98/1 14) usuárias de TARV e 77,7\% (84/1 14) com CD4>500/mm³. Relato de parceria fixa em 78,9\% (90/1 14), havendo sorodiscordância para HIV em 41 , 2\% (47/ 1 14), status sorológico do parceiro desconhecido em $21,9 \%$ e o parceiro desconhece que era infectada em 37,7\% (43/1 14). Última gestação não planejada referida por $71,9 \%(82 / 114)$. Engravidaram no último ano 14,9\%, sendo 70,5\% (12/17) não planejadas. Relato de uso atual de AMPD em 64,9\% (74/1 14) com sangramento genital em 48,2\% (55/1 14) e ganho de peso em $67,5 \%(77 / 114)$. $\bigcirc$ uso de condom masculino foi referido por $62,2 \%(71 / 114)$. Três usuárias de condom feminino sempre e dez eventualmente. Tinham sexo desprotegido vaginal 37,7\% (43/1 14) e anal, 32,4\% (37/ 1 14). Relato de resistência do parceiro para usar preservativo em 30,7\% (35/ 1 14). A dupla contracepção com AMPD e condom foi relatada por 42,9\% (49/1 14). Resistência do parceiro para usar condom foi associada com má adesão (RP=0,3; IC95\% 0,2-0,7; $p<0,001)$. Parceiro desconhecer a infecção da parceira pelo HIV favoreceu a adesão (RP=1,8; IC95\% 1,2-2,7; p=0,013). CONCLUSÃO: Neste estudo, adesão à dupla contracepção com AMPD e condom foi de 42,9\%, mantendo gestações não planejadas e sexo desprotegido. Resistência do parceiro para usar condom aumenta três vezes a chance de a mulher não aderir à dupla proteção e parceiro desconhecer a infecção da mulher quase duplica a chance de ela aderir. Metas: ampliar oferta de novos contraceptivos e envolver parceiros na contracepção e testagem.

\section{Abstract}

PURPOSE: To determine adherence to dual contraception using depot-medroxyprogesterone acetate (DMPA) and condom among HIV-infected women. METHODS: A cross-sectional study carried out from December 2013 to September 2014 at a local reference center, with application of questionnaire elaborated after Delphi panel and content validation to 114 $\mathrm{HIV}(+)$ women aged 15 to 49 years, using DMPA plus condom for contraception. RESULTS: Mean age was 33.2 \pm 7.2 years, mean time since HIV detection was $8.1 \pm 5.2$ years, mean time of antiretroviral use was $6.8 \pm 5$ years and mean CD4 cells $/ \mathrm{mm}^{3}$ count was $737.6 \pm 341$. 1. Sexual HIV acquisition was reported by $98.2 \%$ (1 12/1 14), antiretroviral use by $85.9 \%(98 / 114)$, and $77.7 \%(84 / 114)$ had a CD4>500/ $\mathrm{mm}^{3}$ count. Having a single sex partner was reported by $78.9 \%(90 / 114)$, with HIV serodiscordance in $41.2 \%(47 / 114)$ of couples, $21.9 \%$ did not know the serological
Correspondência

Karina Adami Centro Estadual Especializado em Diagnóstico, Assistência e Pesquisa, Núcleo de Ensino e Pesquisa Rua Comendador José Alves Ferreira, 240, $2^{\circ}$ andar, sala 02 - Garcia CEP: $40100-010$

Salvador (BA), Brasil

Recebido

$06 / 04 / 2015$

Aceito com modificaçõe

$08 / 07 / 2015$
Ambulatório de Ginecologia e Virologia do Centro Estadual Especializado em Diagnóstico, Assistência e Pesquisa da Secretaria Estadual de Saúde do Estado da Bahia - CEDAP/SESAB - Salvador (BA), Brasil.

'Programa de Pós-Graduação em Medicina e Saúde Humana, Escola Bahiana de Medicina e Saúde Humana - Salvador (BA), Brasil. ${ }^{2}$ Grupo Rotinas de Saúde Ampliada - ROSA, Ambulatório de Ginecologia e Virologia, Centro Estadual Especializado em Diagnóstico, Assistência e Pesquisa - CEDAP - Salvador (BA), Brasil.

3Programa de Iniciação Científica, Faculdade de Medicina, Universidade Federal da Bahia - UFBA - Salvador (BA), Brasil. ${ }^{4}$ Centro de Pesquisa, Centro Estadual Especializado em Diagnóstico, Assistência e Pesquisa, Núcleo de Ensino e Pesquisa - Salvador (BA), Brasil.

${ }^{5}$ Curso de Capacitação e Assessoria em Pesquisa Clínica, Centro Estadual Especializado em Diagnóstico, Assistência e Pesquisa, Núcleo de Ensino e Pesquisa - Salvador (BA), Brasil.

Conflito de interesses: não há. 
status of their partner and in $37.7 \%$ of cases $(43 / 114)$ the partner was unaware of the HIV $(+)$ status of the woman. The last pregnancy was unplanned in $71.9 \%$ of cases (82/1 14) and $14.9 \%$ of the women had become pregnant the year before, with pregnancy being unplanned in $70.5 \%(12 / 17)$ of cases. Current use of DMPA was reported by $64.9 \%$ (74/1 14), with genital bleeding in $48.2 \%(55 / 114)$ and weight gain in $67.5 \%$ (77/1 14$)$. Use of a male condom was reported by $62.2 \%$ of the subjects $(71 / 114)$. Three reported that they always used a female condom and ten that they eventually used it. Unprotected vaginal sex was reported by $37.7 \%$ (43/1 14) and unprotected anal intercourse was reported by 32.4\% (37/1 14). Partner resistance to use a condom occurred in 30.7\% of cases (35/1 14). Dual contraception using DMPA with condom was reported by 42.9\% (49/1 14). A partner who resisted wearing a condom was associated with poor adhesion (PR=0.3; $95 \% \mathrm{Cl} 0.2-0.7 ; p<0.001)$. A partner who was unaware that a woman was infected with HIV favored adherence (PR=1.8; 95\%Cl 1.2-2.7; $p=0.013)$. CONCLUSION: The percentage of dual contraception using DMPA plus condom was $42.9 \%$, maintaining unplanned pregnancies and unprotected sex. Resistance of partners to use a condom increased three times the chance of a woman not adhering to dual contraception, and the partner not knowing women's HIV infection almost doubled the chance to adhere to safe contraception. Goals: to offer new hormonal contraceptives and to involve the partners in contraception and serologic detection tests.

\section{Introdução}

A morbimortalidade e o perfil epidemiológico das pessoas infectadas pelo vírus da imunodeficiência humana (HIV), responsável pela síndrome da imunodeficiência adquirida (AIDS), vêm se modificando nas últimas décadas $^{1}$. Nesse período, houve aumento da sobrevida de pessoas vivendo com HIV/AIDS (PVHA) quando em uso de terapias antirretrovirais e no número de mulheres contaminadas pelo vírus em virtude do processo de feminilização da pandemia ${ }^{2}$. O uso de métodos hormonais que sejam compatíveis com uso das terapias antirretrovirais e que possam trazer melhorias clínicas associadas ao uso regular de condom como método de barreira é o desejável ${ }^{3}$. A adesão à dupla contracepção permanece um desafio para a população geral, grupos vulneráveis e entre pessoas infectadas por doença sexualmente transmissível (DST)/HIV/AIDS ${ }^{4,5}$. A taxa de adesão à dupla contracepção referida entre mulheres infectadas pelo HIV gira em torno de $27 \%{ }^{6,7}$, com chance maior que três vezes de engravidar de modo não planejado entre as não usuárias de dupla proteção contraceptiva ${ }^{8}$.

A taxa de eficácia isolada de uso de condom masculino e feminino atribuída na população geral em uso típico é baixa, com percentual alto de falhas contraceptivas, estimado em cerca de 18 a $21 \%$ de mulheres com gestações não planejadas em um ano e descontinuação de seu uso da ordem de 57-59\%?.

A farmacocinética do acetato de medroxiprogesterona de depósito (AMPD) parece não ser afetada pelo uso dos principais esquemas antirretrovirais disponíveis, sendo uma opção contraceptiva frequentemente utilizada por essa população ${ }^{7,10,11}$. O uso de AMPD está relacionado à redução de anemias associadas ao uso de terapias antirretrovirais, em especial nas combinações com AZT (azidotimidina ou zidovudina) ${ }^{6,12}$. Alterações metabólicas como ganho de peso devem ser contornadas com dieta e exercícios físicos regulares, podendo ser atribuídas à droga e melhoradas por mudança comportamental das usuárias e de seu estilo de vida ${ }^{13}$. Recentemente, uma metanálise de estudos observacionais, com 39.560 mulheres na África subsaariana, apontou um aumento na aquisição e transmissão do HIV entre as usuárias de métodos contraceptivos hormonais, em especial o AMPD, que foi associado a $40 \%$ de aumento no risco ${ }^{14}$. Entretanto, não houve evidência clara de causalidade, sendo necessários ensaios clínicos randomizados que eliminem fatores de confusão e estabeleçam que os riscos são maiores que os benefícios ${ }^{15,16}$. Sua eficácia contraceptiva quando em uso habitual é de $6 \%$ de gestações não planejadas em um ano com descontinuação no uso em torno de $44 \%$ na população geral ${ }^{9}$.

Há necessidade de conhecer o perfil da clientela assistida e traçar estratégias para melhorar a adesão às propostas de contracepção segura. Existe a expectativa de diminuir riscos e danos relacionados com a transmissão horizontal e vertical do HIV e outras DSTs através da redução da taxa de gravidez não planejada e uso consistente de condom associado a métodos hormonais em PVHA ${ }^{3,17,18}$. Definir o perfil de manutenção do uso destes métodos contraceptivos, em especial a dupla proteção, se impõe. Essas definições ajudarão a nortear fluxogramas assistenciais no cotidiano dos serviços que assistem a essas pessoas.

O presente trabalho teve o objetivo de verificar a adesão à dupla contracepção entre mulheres infectadas pelo HIV que frequentam um centro de referência, quando em uso de condom e acetato de medroxiprogesterona para fins contraceptivos e, secundariamente, identificar possíveis fatores determinantes da adesão e quantificar o número de gestações não planejadas nessa amostra.

\section{Métodos}

Trata-se de um estudo descritivo, de corte transversal, realizado no centro de referência em DST/HIV/AIDS do Estado da Bahia, o Centro Estadual Especializado em Diagnóstico, Assistência e Pesquisa (CEDAP), no período de dezembro 2013 a setembro 2014. Mulheres assistidas rotineiramente no centro foram convidadas a participar do estudo. Os critérios de inclusão foram: mulheres infectadas 
pelo HIV; em idade reprodutiva de 15 a 49 anos (OMS); expostas ao uso de acetato de medroxiprogesterona e condom para fins contraceptivos entre 2011 e 2013 . Os critérios de exclusão foram: gestantes; histerectomizadas; com laqueadura tubária e parceiro fixo único vasectomizado.

Todas as participantes leram, esclareceram suas dúvidas e assinaram o Termo de Consentimento Livre e Esclarecido (TCLE) antes das entrevistas e o estudo foi aprovado pelo Comitê de Ética em Pesquisa da Secretaria de Saúde do Estado da Bahia (CEP/SESAB) com parecer número 496373 CAAE 19664213.10000.0052.

Foi aplicado questionário construído por painel Delphi, com validação de conteúdo e aperfeiçoamento após aplicação de piloto. Foram entrevistadas 122 mulheres, 15 durante aplicação de questionário piloto e 107 após. Foram excluídas da análise oito entrevistas, sendo quatro por questionários incompletos, duas já submetidas à laqueadura tubária, uma sem vida sexual ativa e uma que usava injetável mensal e não trimestral. Permaneceram para análise 114 entrevistas.

O banco de dados foi construído com o programa EpiData versão 3.1 e analisado com o Statistical Package for the Social Sciences (SPSS) versão 20.0. Utilizados teste do $\chi^{2}$, teste $t$ de Student e de Mann-Whitney quando aplicáveis. Para a análise multivariada, variáveis com $\mathrm{p}<0,20$ na análise univariada ou clinicamente relevantes foram inseridas em modelo de regressão logística, utilizando a técnica backwards. Variáveis que ficaram no modelo final com $\mathrm{p}<0,05$ foram definidas como preditores independentes. A calibração desse modelo foi avaliada pelo teste de Hosmer-Lemeshow. As Odds Ratio foram ajustadas para razão de prevalência pelo método delta.

\section{Resultados}

As mulheres incluídas nessa amostra foram na maioria adultas, com diagnóstico sorológico positivo para o HIV há mais de um ano, adquirido por transmissão sexual, relatando longo período em uso de terapia antirretroviral (TARV), com bom controle da infecção. A maioria era de cor negra, ensino médio completo, renda $\leq 3$ salários mínimos, católica, em relacionamento estável e com filhos, negando uso regular de álcool, cigarro, drogas ou sexo por dinheiro. Há relato de sorodiscordância para o HIV entre os parceiros em mais de um terço da amostra e muitos desconhecem a condição da parceria (Tabela 1).

Dentre as condições obstétricas de interesse, a última gestação foi referida como não planejada por mais de dois terços das mulheres $(71,9 \%$; 82/114), com média de 7,7 76,4 anos para data da última gestação e $8,1 \pm 5,1$ anos de detecção do HIV. No último ano, em regime de dupla contracepção com AMPD e condom, foram
$70,5 \%$ gestações não planejadas (12/17). Em quase um terço das entrevistas, os parceiros ainda queriam filhos.

Setenta e quatro mulheres, aproximadamente $65 \%$, referiam uso atual de AMPD, informando uso correto, em média há mais de 1 ano. A motivação para uso da injeção trimestral ocorreu por indicação médica em 56\% (64/114) e por desejo pessoal em 42,1\% (48/114), podendo ter sido influenciada por ambas as circunstâncias. Destaque para o percentual de mulheres que já haviam descontinuado (35\%; 40/114) e que desejavam suspender o uso do método $(8,7 \% ; 10 / 114)$. Os efeitos colaterais mais frequentes foram sangramento genital $(48,2 \% ; 55 / 114)$ e ganho de peso $(67,5 \% ; 77 / 114)$.

$\mathrm{O}$ uso de condom masculino sempre foi relatado por $62,2 \%$ (71/114), enquanto que o uso de condom feminino foi restrito a 3 usuárias regulares e 10 com uso eventual.

Tabela 1. Características clínicas e epidemiológicas da amostra

\begin{tabular}{|c|c|c|c|c|c|}
\hline Características (n=114) & n & $\%$ & Média & Mediana & $D P I Q$ \\
\hline Idade (anos) & & & 33,2 & & 7,2 \\
\hline $15-19$ & 5 & 4,4 & & & \\
\hline $19-49$ & 109 & 95,6 & & & \\
\hline Negras & 57 & 50,0 & & & \\
\hline Católicus & 49 & 42,9 & & & \\
\hline Escolaridade ( $\leq 8$ anos) & 111 & 97,3 & & & \\
\hline Renda familiar ( $\leq 3$ SM) & 102 & 89,5 & & & \\
\hline Uso de drogas ilícitas & 10 & 8,8 & & & \\
\hline Tabagismo & 26 & 22,8 & & & \\
\hline Uso social de álcool & 59 & 51,7 & & & \\
\hline Sexo por dinheiro & 10 & 8,8 & & & \\
\hline Aquisição sexual do HIV & 112 & 98,2 & & & \\
\hline Tempo de diagnóstico do HIV (anos) & & & 8,1 & & 5,2 \\
\hline Até 1 & 12 & 10,5 & & & \\
\hline Mais que 1 & 102 & 89,5 & & & \\
\hline Usuárias de TARV & 98 & 85,9 & & & \\
\hline Uso de TARV (anos) & & & 6,8 & & 5 \\
\hline CV (cópias/mL) & & & & 4.710 & $1-198$ \\
\hline CV indetectável $(<40)$ & 76 & 66,7 & & & \\
\hline$>40$ & 38 & 33,3 & & & \\
\hline CD4 (células/mm³) & & & 737,6 & & 341,1 \\
\hline$>500$ & 84 & 77,7 & & & \\
\hline$<500$ & 30 & 26,3 & & & \\
\hline Parcerias ao longo da vida & & & & 5 & $3-10$ \\
\hline Idade da primeira relação (anos) & & & 16,1 & & 2,8 \\
\hline Idade da primeira gestação (anos) & & & 20,2 & & 5,7 \\
\hline Número de gestações & & & 2,3 & & 1,7 \\
\hline Parceiro único & 90 & 78,9 & & & \\
\hline Tempo de relação estável & & & 6,4 & & 5,0 \\
\hline Sorodiscordância para HIV & 47 & 41,2 & & & \\
\hline Parceiro desconhece infecção da mulher & 43 & 37,7 & & & \\
\hline Parceiro resiste em usar condom & 35 & 30,7 & & & \\
\hline
\end{tabular}

DP: desvio padrão; IC: intervalo interquartil; SM: salário mínimo; TARV: terapia antirretroviral; $\mathrm{CV}$ : carga viral. 
Dentre as usuárias de condom masculino, muitas afirmaram fazer uso regular, mas chama a atenção o uso irregular de condom nas práticas sexuais com sexo desprotegido vaginal $(37,7 \%$; 43/114), anal $(32,4 \% ; 37 / 114)$ e oral $(63,1 \% ; 72 / 114)$.

A adesão à dupla proteção contraceptiva nessa amostra representou mais que um terço da amostra (Tabela 2). Nenhum dado sociodemográfico descrito neste estudo se revelou significante para predizer adesão à dupla contracepção com AMPD e condom. Permaneceram significantes como preditores dois aspectos relacionados aos parceiros. O relato de resistência do parceiro para usar condom apresentou-se como fator associado à não adesão a essa dupla contracepção, com chance 3 vezes maior de não aderir $(\mathrm{OR}=0,1 ; \mathrm{IC} 95 \% 0,04-0,42 ; \mathrm{RP}=0,3$; IC95\% 0,2-0,7). Quando o parceiro desconhece que a mulher é infectada pelo HIV, existe uma chance de quase 2 vezes mais de a mulher aderir à dupla contracepção referida $(\mathrm{OR}=3,2$; IC95\% 1,3-8,1; RP=1,8; IC95\% 1,2-2,7) (Tabela 3).

Tabela 2. Prevalência de uso de condom, acetato de medroxiprogesterona e de ambos (dupla contracepç̃̃o)

\begin{tabular}{lcc}
\hline & n $(\%)$ & $\begin{array}{c}\text { IC } \\
(\%)\end{array}$ \\
\hline AMPD & $74(64,9)$ & $55-73$ \\
Condom sempre* & $71(62,2)$ & $52-71$ \\
AMPD + condom sempre & $49(42,9)$ & $34-52$ \\
\hline
\end{tabular}

*Condom masculino sempre (nenhuma usuária de condom feminino da amostra se manteve usando AMPD).

IC: intervalo de confiança calculado pelo teste exato de Fisher; AMPD: acetato de medroxiprogesterona.

Tabela 3. Preditores de adesão à dupla contracepção com condom e acetato de medroxiprogesterona na amostra

\begin{tabular}{|c|c|c|c|}
\hline \multirow[t]{2}{*}{ Características } & $\begin{array}{l}\text { Adesão } \\
(\mathrm{n}=49)\end{array}$ & $\begin{array}{c}\text { Não adesão } \\
(n=65)\end{array}$ & \multirow{2}{*}{ Valor $\mathrm{p}$} \\
\hline & \multicolumn{2}{|c|}{ Média (DP) } & \\
\hline Idade (anos) & $34(7,2)$ & $32(7,2)$ & 0,17 \\
\hline Usuárias de TARV & $45(92)$ & $53(81)$ & 0,06 \\
\hline Renda familiar ( $\leq 3$ salários mínimos) & $47(96)$ & $55(85)$ & 0,05 \\
\hline Tabagismo & $9(18)$ & $4(6,1)$ & 0,12 \\
\hline $\begin{array}{l}\text { Parceiro desconhece sua condição de } \\
\text { infectada pelo HIV }\end{array}$ & $22(45)$ & $21(32)$ & 0,06 \\
\hline Parceiro resiste em usar condom & $7(14)$ & $28(43)$ & 0,002 \\
\hline Preditores & $\begin{array}{c}\text { OR } \\
(\mathrm{ICP5 \% )} \\
\end{array}$ & $\begin{array}{c}\mathrm{RP} \\
(\mathrm{IC95 \% )} \\
\end{array}$ & Valor $p$ \\
\hline $\begin{array}{l}\text { Parceiro desconhece sua condição de } \\
\text { infectada pelo HIV }\end{array}$ & $3,2(1,3-8,1)$ & $1,8(1,2-2,7)$ & 0,013 \\
\hline Parceiro resiste em usar condom & $0,1(0,04-0,42)$ & $0,3(0,2-0,7)$ & $<0,001$ \\
\hline \multicolumn{4}{|c|}{$\begin{array}{l}\text { *Primeira análise univariada: incluídas na análise multivariada por } p<0,20 \text { na } \\
\text { análise do } \chi^{2} \text { de Pearson; ** segunda análise multivariada: preditores considerados } \\
\text { com } p<0,05 \text { na regressão logística por backwards com calibração (teste de } \\
\text { Hosmer-Lemeshow) - } p=0,92 \text { e ajuste da OR para RP por método delta. }\end{array}$} \\
\hline \multicolumn{4}{|c|}{$\begin{array}{l}\text { DP: desvio padrão; TARV: terapia antirretroviral; OR: Odds Ratio; IC95\%: intervalo } \\
\text { de confiança de 95\%; RP: razão de prevalência. }\end{array}$} \\
\hline
\end{tabular}

\section{Discussão}

O achado mais importante do presente estudo foi o percentual de adesão à dupla contracepção maior que o relatado na literatura, sem destaque para nenhum caráter sociodemográfico como preditor ${ }^{17,18}$.

A taxa de gestação não planejada nessa amostra foi semelhante à de populações incluídas em outros estudos com proporção estimada de $62 \%$ numa coorte africana e 83,1\% entre puérperas infectadas pelo HIV em corte transversal também na África ${ }^{8,19}$. Isso alerta para o fato de que, mesmo nessa amostra de conveniência, com acesso a informações, aos insumos sugeridos e à equipe multidisciplinar, ainda existe um grupo que não adere às práticas de contracepção e sexo seguros, apresentando alta taxa de gestação não planejada.

O sexo desprotegido chama a atenção nesse estudo apresentando um percentual elevado de pessoas expostas ao risco, em especial quando se nota a parcela de casais sorodiscordantes. Clinicamente, existe o risco de transmissão para parcerias negativas, mas também há a preocupação com a superinfecção entre os casais soroconcordantes, que corrobora maior resistência viral à TARV, e ainda a aquisição de outras DSTs, prejudicando ou interferindo na evolução natural das doenças HIV-relacionadas ${ }^{12,15,20,21}$. O percentual de sexo anal desprotegido é um dado preocupante. Segundo o Centers for Disease Control and Prevention (CDC $)^{22}$, de cada $10 \mathrm{mil}$ relações sexuais, ocorrem 138 infecções no sexo anal receptivo para 11 no sexo anal insertivo, 8 no sexo vaginal receptivo e 4 no sexo vaginal insertivo. Em uma metanálise de 2010, Baggaley et al..$^{23}$ estimaram que o risco de adquirir o HIV através do sexo anal é 18 vezes maior que no vaginal. $\mathrm{Na}$ abordagem do risco, após sexo desprotegido, deve-se atentar para a necessidade de o casal e parcerias realizarem testagens sorológicas para infecção sexualmente transmitida (IST) e realizar a profilaxia pós-exposição sexual quando houver sorodiscordância para o HIV ${ }^{24,25}$.

$\mathrm{Na}$ última versão do "Protocolo Clínico e Diretrizes Terapêuticas para Manejo da Infecção pelo HIV em Adultos”, publicado em 2014 pelo Ministério da Saúde Brasileiro (Secretaria de Vigilância em Saúde/ Departamento de DST, AIDS e Hepatites Virais), já há recomendação de início precoce da TARV para tornar a carga viral indetectável e reduzir as taxas de transmissão horizontal ${ }^{25}$. Evidências atuais demonstram o impacto da TARV nessa redução para cerca de três vezes menos transmissão entre usuários regulares de TARV $^{26}$. Segundo estimativas de Del Romero et al. ${ }^{27}$, a transmissão horizontal chega a zero para casais sorodiscordantes quando o sujeito positivo para o HIV usa regularmente os antirretrovirais, tem carga viral 
indetectável por mais de seis meses e não tem outras DSTs. Mas basta adquirir uma nova DST ou alguns dias sem uso da TARV para que o quadro se modifique e volte a transmitir, com taxa estimada em $8,6 \%$ sem intervenções. A via sexual sem barreiras de proteção continua promovendo a aquisição de outras doenças como hepatites B e C, sífilis, Chlamydia trachomatis, Neisseria gonorrboea, Mycoplasma hominis, Ureaplasma urealyticum, papilomavírus humano, entre outros ${ }^{21}$. Permanece o desafio do sexo seguro universal, que, por sua vez, perpassa uma série de modificações culturais e estruturais que o tornam distante ${ }^{5,28,29}$.

$\mathrm{O}$ uso e manutenção da injeção trimestral nessa amostra foram superiores às estimativas da população geral e de outras casuísticas da literatura em populações relacionadas ao $\mathrm{HIV}^{12,30,31}$. A continuidade de uso do AMPD na população geral é citada em torno de $56 \%$, segundo dados da Joint United Nations Programme on HIV/ $A I D S^{17}$; em amostra de mulheres soropositivas para o HIV em um site inglês, foi de $40 \%$ e em publicação africana, $15 \%{ }^{15,19}$. Numa revisão da Cochrane em 2013, envolvendo trabalhos desenvolvidos em populações africanas, totalizando 8.882 mulheres infectadas pelo HIV, observou-se maior descontinuidade do uso de hormônios contraceptivos nessas mulheres quando comparadas a mulheres soronegativas para HIV, quase 3 vezes mais $(\mathrm{OR}=2,52 \text {; IC95\% 1,53-4,14) })^{32}$.

$A$ adaptação ao uso de condom feminina é uma barreira citada constantemente nos trabalhos sobre contracepção e sexo seguro, figurando sempre com baixas taxas de uso, mesmo entre infectados pelo HIV $^{28}$. Nesta casuística, apenas três usuárias mantinham o método cotidianamente, a despeito de, no centro onde ocorreu o estudo, terem sido distribuídas 8.831 unidades de preservativo feminino entre 2011 e 2014, segundo controle de dispensação da farmácia da unidade. Mesmo promovendo uma proteção mais ampla às ISTs pela barreira gerada ao cobrir o vestíbulo vulvar, o condom feminino se limita ao uso no sexo vaginal. As práticas de sexo oral e anal exigem outros materiais de proteção, sendo adequado o uso de condom masculino. Portanto, mesmo que o condom feminino fomente o empoderamento da mulher no sexo, ele se limita ao sexo convencional. Na prática, há que se ter flexibilidade e olhar individualizado para utilização do material de barreira disponível e que contemple as demandas individuais.

$\mathrm{Na}$ elaboração de uma linha de cuidado, o envolvimento da parceria tem sido cada vez mais relevante ${ }^{2,18,25}$. Neste estudo, a população apresentou maior percentual de parceria fixa única e um terço de sorodiscordância. O único preditor isolado de má adesão à dupla contracepção foi a resistência do parceiro em usar condom e as mulheres aderiram mais quando seus parceiros desconheciam que era infectada pelo HIV. Na assistência ao HIV, assim como frente às demais DSTs, classicamente, a abordagem deve assegurar terapia, testagens e aconselhamento sexual às parcerias.

Existe uma limitação para análises, na medida em que a amostra é composta de usuárias que aderem ao serviço e que estão em seguimento regular, com bom controle de doença HIV-relacionada. Na literatura, há uma percepção de que determinados grupos populacionais tenham maior tendência a não aderir às propostas contraceptivas, como adolescentes, usuárias de drogas, pessoas de baixa renda e escolaridade, aquelas com diagnóstico recente para o HIV e aquelas com dificuldades de acesso aos insumos e serviços de saúde $e^{20,21,30}$. Grupos de maior vulnerabilidade não foram contemplados nessa amostra, apontando uma necessidade de ampliação de seu acesso ao serviço.

Este estudo se propôs a determinar a adesão à dupla contracepção entre mulheres HIV-positivas, usando acetato de medroxiprogesterona e condom, num centro de referência em DST/HIV/AIDS. Seu cálculo amostral inicial se baseou nesse objetivo, não havendo a pretensão de ter poder para detectar associações entre determinantes e a adesão ou não. Muitas das variáveis testadas não se mostraram associadas ao desfecho principal, mas talvez outros preditores se destacassem caso a amostra fosse ampliada. Caberia ainda uma coorte com seguimento temporal dos eventos.

Em conclusão, a prevalência da adesão à dupla contracepção, proposta num centro de referência, realizada pela combinação dos métodos acetato de medroxiprogesterona de depósito trimestral e condom, entre mulheres infectadas pelo HIV, foi maior que o relatado em outros estudos, porém aquém das expectativas para sua efetiva proteção contraceptiva, com taxas altas de falhas contraceptivas e gestação não planejada. A oferta de novas opções contraceptivas, por exemplo, os métodos reversíveis de longa duração (LARC), poderá colaborar para o aumento da adesão e eficácia contraceptivas nessa população, assim como ocorre na população geral ${ }^{33-35}$. A resistência do parceiro em usar condom foi um preditor, com chance três vezes maior de má adesão à dupla contracepção e o desconhecimento do parceiro quanto à condição da mulher ser soropositiva para o HIV como determinante de maior adesão com chance quase que duas vezes maior de aderir à dupla contracepção. O envolvimento das parcerias nas escolhas contraceptivas, testagens sorológicas, estratégias de profilaxia e prevenção, assim como aumento da oferta de contraceptivos são metas a serem alcançadas, objetivando aumento da adesão contraceptiva e redução da taxa de gestações não planejadas, transmissão vertical e horizontal do vírus. 
1. World Health Organization. Chronic HIV care with ARV therapy and prevention. Geneva: WHO; 2007.

2. Boletim Epidemiológico - HIV-AIDS [Internet]. Brasília (DF): Ministério da Saúde/Secretaria de Vigilância em Saúde/Programa Nacional de DST e AIDS. 2014 [citado 2015 Mar 3];3(1). Disponível em: <http://www.aids.gov.br/sites/default/files/anexos/ publicacao/2014/56677/boletim_2014_final_pdf_15565.pdf>

3. World Health Organization. Policy implications: programmatic and research considerations for hormonal contraception for women at risk of HIV and women living with HIV. Geneva: WHO; 2012.

4. Lawani LO, Onyebuchi AK, lyoke CA. Dual method use for protection of pregnancy and disease prevention among HIV-infected women in South East Nigeria. BMC Womens Health. 2014;14(1):39.

5. Sanders SA, Yarber WL, Kaufman EL, Crosby RA, Graham CA, Milhausen RR. Condom use errors and problems: a global view. Sex Health. 2012;9(1):81-95.

6. Robinson JA, Jamshidi R, Burke AE. Contraception for the HIV-positive woman: a review of interactions between hormonal contraception and antiretroviral therapy. Infect Dis Obstet Gynecol. 2012;2012:890160.

7. Amaral E, Viscola MA, Bahamondes L. [Hormonal contraception and antiretroviral therapy among HIV-infected women]. Rev Bras Ginecol Obstet. 2006;28(1 1):680-4. Portuguese.

8. Schwartz SR, Rees H, Mehta S, Venter WD, Taha TE, Black V. High incidence of unplanned pregnancy after antiretroviral therapy initiation: findings from a prospective cohort study in South Africa. PLoS One. 2012;7(4):e36039.

9. Trussell J. Contraceptive failure in the United States. Contraception. $2011 ; 83(5): 397-404$

10. Nanda K, Amaral E, Hays M, Viscola MM, Mehta N, Bahamondes L. Pharmacokinetic interactions between depot medroxyprogesterone acetate and combination antiretroviral therapy. Fertil Steril. 2008;90(4):965-71 .

11. Vieira CS, Bahamondes MV, Souza RM, Brito MB, Prandini TR, Amaral $E$, et al. Effect of antiretroviral therapy including lopinavir/ritonavir or efavirenz on etonogestrel-releasing implant pharmacokinetics in HIVpositive women. J Acquir Immune Defic Syndr. 2014;66(4):378-85.

12. Heikinheimo $O$, Lähteenmäki P. Contraception and HIV infection in women. Hum Reprod Update. 2009; 15(2): 165-76.

13. Modesto W, Santos PN, Correia VM, Borges L, Bahamondes L. Weight variation in users of depot-medroxyprogesterone acetate, the levonorgestrel-releasing intrauterine system and a copper intrauterine device for up to ten years of use. Eur J Contracept Reprod Health Care. 2015;20(1):57-63.

14. Polis CB, Phillips SJ, Curtis KM, Westreich DJ, Steyn PS, Raymond E, et al. Hormonal contraceptive methods and risk of HIV acquisition in women: a systematic review of epidemiological evidence. Contraception. 2014;90(4):360-90.

15. Morrison CS, Richardson BA, Mmiro F, Chipato T, Celentano DD, Luoto J, et al. Hormonal contraception and the risk of HIV acquisition. AIDS. 2007;21(1):85-95.

16. Heffron R, Donnell D, Rees H, Celum C, Mugo N, Were E, et al. Use of hormonal contraceptives and risk of HIV-1 transmission: a prospective cohort study. Lancet Infect Dis. 2012;12(1):19-26.

17. Joint United Nations Programme on HIV/AIDS. Global AIDS response progress reporting 2014: construction of core indicators for monitoring the 2011 United Nations Political Declaration on HIV and AIDS. Geneva: UNAIDS; 2014.

18. Walensky RP, Kuritzkes DR. The impact of the President's Emergency Plan for AIDS Relief (PEPfAR) beyond HIV and why it remains essential. Clin Infect Dis. 2010;50:272-5.
19. Duncan S, Hawkins F, Desmond N. Postnatal contraceptive choices among women living with HIV: a decade of experience in a community-based integrated sexual health clinic. J Fam Plan Reprod Health Care. 2012;39(1):17-20.

20. Huijbregts RP, Helton ES, Michel KG, Sabbaj S, Richter HE, Goepfert $P$, et al. Hormonal contraception and HIV-1 infection: medroxyprogesterone acetate suppresses innate and adaptive immune mechanisms. Endocrinology. 2013;154(3):1282-95.

21. Travassos AG, Brites C, Netto EM, Fernandes SA, Rutherford GW, Queiroz CM. Prevalence of sexually transmitted infections among HIV-infected women in Brazil. Braz J Infect Dis. 2012;16(6):581-5.

22. Centers for Disease Control and Prevention (CDC). Update to $C D C^{\prime}$ 's U.S. medical eligibility criteria for contraceptive use, 2010 : revised recommendations for the use of hormonal contraception among women at high risk for HIV infection or infected with HIV. MMWR Morb Mortal Wkly Rep. 2012;61 (24):449-52.

23. Baggaley RF, White RG, Boily MC. HIV transmission risk through anal intercourse: systematic review, meta-analysis and implications for HIV prevention. Int J Epidemiol. 2010;39(4):1048-63.

24. Vieira CS. Protocolo municipal de saúde reprodutiva para pessoas que vivem e convivem com HIV/AIDS. Ribeirão Preto: Faculdade de Medicina de Ribeirão Preto, Universidade de São Paulo; 2013.

25. Brasil. Ministério da Saúde. Secretaria de Vigilância em Saúde [Internet]. Protocolo clínico e diretrizes terapêuticas para manejo da infecção pelo HIV em adultos. Brasília (DF): Ministério da Saúde; 2013 [citado 2014 Dez 12]. Disponível em: <http://www.aids. gov.br/sites/default/files/anexos/publicacao/2013/55308/ protocolo_13_3_2014_pdf_28003.pdf>

26. Anglemyer A, Rutherford GW, Baggaley RC, Egger M, Siegfried $\mathrm{N}$. Antiretroviral therapy for prevention of HIV transmission in HIVdiscordant couples. Cochrane Database Syst Rev. 2011 ; (8):CD009153.

27. Del Romero J, Río I, Castilla J, Baza B, Paredes V, Vera M, et al. Absence of transmission from HIV-infected individuals with HAART to their heterosexual serodiscordant partners. Enferm Infecc Microbiol Clin. 2014 Dec 30. [Epub ahead of print].

28. Magalhães J, Rossi AD, Amaral E. [Female condom use among HIV-infected women]. Rev Bras Ginecol Obstet. 2003;25(6):38995. Portuguese.

29. Lopez LM, Otterness C, Chen M, Steiner M, Gallo MF. Behavioral interventions for improving condom use for dual protection. Cochrane Database Syst Rev. 2013;10:CD010662.

30. Magalhães J, Amaral E, Giraldo PC, Simões JA. HIV infection in women: impact on contraception. Contraception. 2002;66(2):87-91.

31. Nanda K, Morrison CS, Kwok C, Byamugisha J, Jones L, Sriplienchan S, et al. Discontinuation of oral contraceptives and depot medroxyprogesterone acetate among women with and without HIV in Uganda, Zimbabwe and Thailand. Contraception. 2011;83(6):542-8.

32. Lopez LM, Hilgenberg D, Chen M, Denison J, Stuart G. Behavioral interventions for improving contraceptive use among women living with HIV. Cochrane Database Syst Rev. 2013;1:CD010243.

33. Whiteman MK, Kissin DM, Samarina A, Curtis KM, Akatova N, Marchbanks $P$, et al. Determinants of contraceptive choice among women with HIV. AIDS. 2009;23(Suppl 1):S47-54.

34. Winner B, Peipert JF, Zhao Q, Buckel C, Madden T, Allsworth $\mathrm{JE}$, et al. Effectiveness of long-acting reversible contraception. $\mathrm{N}$ Engl J Med. 2012;366(21):1998-2007.

35. Pittman ME, Secura GM, Allsworth JE, Homco JB, Madden T, Peipert JF. Understanding prescription adherence: pharmacy claims data from the Contraceptive CHOICE Project. Contraception. 2011 ; 83(4):340-5. 